\title{
EXPERIMENTAL ANALYSIS AND THERMAL COMFORT INDEX OF AIR CONDITIONED MEETING HALL
}

\author{
Daniel Lawrence $I^{1}$, Jayabal $S^{2}$, Rajmohan . ${ }^{3}$, Thiruneelakandan $G^{4}$ \\ ${ }^{I}$ Faculty, Department of Mechanical Engineering, Anna University Regional Office Madurai, Madurai, Tamilnadu \\ ${ }^{2}$ Faculty, Department of Mechanical Engineering, Alagappa chettiar college of engineering and Technology, \\ Karaikudi, Tamilnadu \\ ${ }^{3}$ Faculty, Department of Mechanical Engineering, Anna University Regional Office Madurai, Madurai, Tamilnadu \\ ${ }^{4}$ Faculty, Department of Mechanical Engineering, Anna University Regional Office Madurai, Madurai, Tamilnadu
}

\begin{abstract}
India's building energy consumption is increasing continuously. The subcontinent does not have custom made thermal comfort standards. We conducted thermal comfort field study in meeting hall, Madurai, tamilnadu, india. Experimental study of thermal comfort was conducted in three levels of load conditions and three levels of air flow in meeting hall during summer 2014. The above three level of load condition and air flow measurements values observed by using direct reading instruments. Thermal comfort indices were also calculated and compare to the natural ventilated meeting hall and outdoor environment. This study aims to evaluate the indoor comfort quality of the meeting hall. This paper is focused on improving the indoor air quality by controlling the vital parameters like carbon dioxide, carbon monoxide, oxygen, room temperature, relative velocity and relative humidity which were used as indicators for indoor air quality and comfort levels. The investigated meeting hall building is located in India, tamilnadu, Madurai and the volume of the building is about $10.60 \mathrm{~m}$ (length) $\times 9.14 \mathrm{~m}$ (width) $\times 3 \mathrm{~m}$ (height). This paper provides the detailed specifications to improve the thermal comfort in the air conditioned living atmosphere and ensure safety and healthy living environment. This paper contained Experimental analysis and Calculation of thermal comfort index.
\end{abstract}

Keywords: Meeting hall, Indoor Air Quality, various loads, various air flow rates, thermal comfort index.

\section{INTRODUCTION}

The national building code of India and ASHARE standard 55 specifies two narrow range of indoor comfort temperatures $\left(21^{\circ} \mathrm{C}-26^{\circ} \mathrm{C}\right)$ irrespective of building type or location of environment. Indian heating ventilation and air conditioning designers predominantly make use of fanger predicted mean vote (PMV) model to design the indoor environments.

T.T.Chowa at el. Conducted result analysis shows that, like in many other Asian cities, the thermal sensation of the Hong Kong people is sensitive to air temperature and speed, but not much to humidity. With bodily air speed at $0.1-0.2$ $\mathrm{m} / \mathrm{s}$, clothing level $0.55 \mathrm{clo}$ and metabolicrate $1 \mathrm{met}$, the neutral temperature was found around $25.4^{\circ} \mathrm{C}$ for sedentary working environment [1]. The outdoor level of carbondioxide is usually $350-450$ parts per million (ppm). The carbon-dioxide level is usually greater inside a building than outside. If the indoor carbon-dioxide level is more than 1000 $\mathrm{ppm}$, when there is inadequate ventilation, there may be health implications and the occurrence of physical conditions such as headache, fatigue, and irritation of the eyes and the throat [2]. Carbon-monoxide is colorless and odourless, and is a normal constituent of exhaust gases from incomplete combustion. $\mathrm{CO}$ is dangerous (more so than $\mathrm{CO}_{2}$ ) because it inhibits the blood's ability to carry oxygen to vital organs such as the heart and brain. For office areas, levels of carbon-monoxide are normally between 0 and 5 $\mathrm{ppm}$. Concentrations greater than $5 \mathrm{ppm}$ indicates the possible presence of exhaust gases in the indoor environment and should be investigated. According to the ASHRAE standard, levels of carbon-monoxide inside buildings should not exceed $9 \mathrm{ppm}$. If the CO level inside a building is detected above $100 \mathrm{ppm}$, the building should be evacuated until the source is identified and the situation is corrected [2]. The ASHRAE guideline is that indoor temperatures in the winter are maintained between $20^{\circ} \mathrm{C}$ to $24^{\circ} \mathrm{C}$. Temperature in the summer should be maintained between $22.8^{\circ} \mathrm{C}$ to $26.1^{\circ} \mathrm{C}$ [2]. Mohammad Taleghani at el. Discussed the main result of the field studies, three internationally well known thermal comfort standards: ASHRAE55-2010, EN15251:2007 and ATG were comprehensively presented. In each standard, database, basic equations, upper and lower boundaries and reference temperatures were discussed comprehensively [3]. M. Kavgic at el. Analyzed the level of indoor air quality and thermal comfort in a typical medium-sized mechanically ventilated theatre, and to identify where improvements could typically be made, a comprehensive post-occupancy evaluation study was carried out on a theatre in Belgrade [5].

K. Luck. Explain the main emphasis in this case is on the well-being of humans, promoting their performance and hence their efficiency, and on achieving high exergetic efficiency [6]. 
Frauke Oldewurtel at el. Presented this paper an MPC controller, which controls the building based on a standard fixed occupancy schedule, is used as a benchmark. The energy use of this benchmark is compared with three other control strategies [8]. Pawel Wargocki at el. Presented this paper is an overall summary of research by the authors on how classroom conditions affect the performance of schoolwork by children, motivated by the fact that the thermal and air quality conditions in school classrooms are now almost universally worse than the relevant standards and building codes stipulate that they should be[10]. Above papers, a meeting hall with air conditioning facility was selected and experiments were conducted to study the characteristics of IAQ likely carbon dioxide, carbon monoxide, oxygen, room temperature, air velocity and relative humidity which were used as indicators for IAQ and comfort levels in this present investigation.

\section{METHODOLOGY}

Investigate the air conditioning systems in modern buildings like meeting hall. The Variable parameters of air conditioning Systems are Air Exchange rate, Air velocity, Thermal properties of indoor Equipments and human. The Measurable parameters of air conditioning systems are Air flow, Relative humidity, Temperature and Concentration of Gaseous pollutants and Oxygen. These parameters are to be continuously monitored by an instrument and simulation by using various ventilation systems and have to be calibrated before Experimental measurement.

\subsection{Field Study}

The experiment was carried out in meeting hall in office building. Measurement data in variable volume rate the room should be in various climatic environments. The measurable parameters are air flow, temperature flow, gas flow and relative humidity was measured every 30 mints in natural ventilation and air conditioning (4 individual duct ) was running 8 hours each case. Also outdoor climate condition was measured every half hour once. Indoor conditions the measurement was taken Middle of the room. Were found regarding the current indoor environment of indoor particulate are mentioned below table in field studies.

Table 2.1 Material Used In Indoor Environment

\begin{tabular}{|l|l|l|}
\hline S No & Load & volume \\
\hline 1 & Tube light & $8(40 \mathrm{w})$ \\
\hline 5 & Roofing & concrete \\
\hline 6 & Flooring & Marble (Thickness $10 \mathrm{~cm})$ \\
\hline 7 & Concrete Wall & 4 side (Thickness $15 \mathrm{~cm})$ \\
\hline 9 & Load & 45 person \\
\hline 10 & Fresh air supply & $10 \%$ \\
\hline
\end{tabular}

\subsection{Outdoor Environment}

The outdoor measurements were performed in this experiment. Morning to evening reported there thermal comfort. The six parameters are observed in this experiment was completed in eight days for each case two times. The parameters describe the quality of outdoor environment.

\subsection{Description of the Problem}

- To evaluate indoor air quality (IAQ) factors in three level of air flow volume, three level of load and three level of time period.

- $\quad$ Predict the thermal comfort in meeting hall

\subsection{Objective}

- To provide better indoor air quality (IAQ) performance to the Occupant.

- To satisfy the all level of expectation on thermal comfort in meeting hall.

- Also eliminate the negative influence upon the human health issue and Adaptive for variable climatic environments.

\subsection{Experimental Approach}

Investigate the air conditioning systems in modern building like meeting hall.

The Variable parameters of air conditioning Systems are Air flow rate, about Human load with respect to time period.

The Measurable parameters of air conditioning systems are Relative velocity, Relative humidity, indoor Temperature, Carbon dioxide, Carbon monoxide and Oxygen.

These parameters are continuously monitored by direct reading instrument has to be calibrated before Experimental measurement.

Table 2.6 ASHRAE standard parameters for indoor environment $^{[2]}$

\begin{tabular}{|l|l|l|}
\hline $\begin{array}{l}\text { S. } \\
\text { No }\end{array}$ & Parameter & ASHRAE standard \\
\hline 1 & Carbon-dioxide & 0 to1000ppm \\
\hline 2 & Carbon-monoxide & 0 to9ppm \\
\hline 3 & Temperature & $20^{\circ} \mathrm{C}$ to $26.1^{\circ} \mathrm{C}$ \\
\hline
\end{tabular}

\subsection{Instruments used for Measurements}

- Digital Thermo Hygrometer (Range: Temp $-50^{\circ} \mathrm{C}$ to $+70^{\circ} \mathrm{C}$, RH $10 \%$ to $99 \%$, Accuracy: Temp $\pm 1^{\circ} \mathrm{C}, \mathrm{RH}$ $\pm 5 \%$ )

- Anemometer (Range: 0.4-45 m/sec, Accuracy: $\pm 2 \%+0.1 \mathrm{~m} / \mathrm{s}$ )

- CO Meter (Range: 0 to1000ppm, Accuracy : $\pm 5 \%+2 \mathrm{ppm})$

- $\mathrm{CO}_{2}$ Meter (Range: 0 to 4000ppm, Accuracy : $\pm 40 \mathrm{ppm})$

- $\mathrm{O}_{2}$ Meter (Range: 0 to $30 \%$, Accuracy: $\pm 1 \%+0.2 \%$ ) 


\subsection{Measurement observation}

Experimental Measurements are noted at three level of air flow volume with sixty human load with furniture's on the one full working hours. The measurable parameters are temperature, relative humidity, relative velocity, carbon dioxide, carbon monoxide and oxygen are continuously measured eight hours time period with 30 mints interval air conditioned environment in meeting hall. Because of urban environment the outdoor environment measurable parameters of the above are measured every half hour once. All the measurable parameters are measured in indoor was taken Middle of the room.

Experimental measurements are presented in table's representation of Air flow volume $1400 \mathrm{cfm}$ with human load.

\section{RESULT \& DISCUSSION}

\subsection{Experimental Readings for Meeting Hall:}

The experimental indoor air quality results are presented in table. Experimental measurements are presented in table's representation of Air flow volume $1400 \mathrm{cfm}$ with human load.

Table 3.1 Airflow volume 1400 with without loading

\begin{tabular}{|c|c|c|c|c|c|c|c|}
\hline $\begin{array}{l}\text { Tim } \\
\mathrm{e}\end{array}$ & $\begin{array}{l}\text { Indoor } \\
/ \\
\text { Outdo } \\
\text { or }\end{array}$ & $\begin{array}{l}\text { Tem } \\
\mathrm{p} \\
\left({ }^{\circ} \mathrm{C}\right)\end{array}$ & $\begin{array}{l}\text { RH } \\
(\% \\
)\end{array}$ & $\begin{array}{l}\mathrm{CO} \\
\text { (pp } \\
\mathrm{m})\end{array}$ & $\begin{array}{l}\mathrm{CO}_{2} \\
\text { (pp } \\
\mathrm{m})\end{array}$ & $\begin{array}{l}\mathrm{O}_{2} \\
(\%)\end{array}$ & $\begin{array}{l}\mathrm{RV} \\
(\mathrm{m} / \mathrm{s} \\
)\end{array}$ \\
\hline \multirow[b]{2}{*}{9.00} & ID & 29.6 & 56. & 0.0 & 433 & 19. & 0.40 \\
\hline & OD & 33.2 & 50. & 1.0 & 392 & 19. & 1.50 \\
\hline \multirow[b]{2}{*}{9.30} & ID & 25.9 & 49. & 0.0 & 436 & 18. & 0.40 \\
\hline & OD & 35.5 & 47. & 1.0 & 383 & 19. & 1.70 \\
\hline \multirow[b]{2}{*}{10.0} & ID & 24.4 & 46. & 0.0 & 427 & 19. & 0.40 \\
\hline & OD & 37.0 & 45. & 1.0 & 361 & 19. & 1.20 \\
\hline \multirow[b]{2}{*}{10.3} & ID & 24.2 & 48. & 0.0 & 439 & 19. & 0.40 \\
\hline & OD & 37.5 & 45. & 2.00 & 364 & 19. & 1.20 \\
\hline \multirow[b]{2}{*}{11.0} & ID & 23.1 & 49. & 0.00 & 452 & 18. & 0.40 \\
\hline & OD & 38.0 & 46. & 0.00 & 382 & 19. & 0.70 \\
\hline \multirow[b]{2}{*}{11.3} & ID & 22.0 & 50. & 0.00 & 443 & 18. & 0.40 \\
\hline & OD & 39.0 & 44. & 0.00 & 372 & 19. & 1.70 \\
\hline \multirow[b]{2}{*}{12.0} & ID & 22.9 & 49. & 0.00 & 429 & 18. & 0.40 \\
\hline & OD & 39.5 & 36. & 0.00 & 345 & 20. & 0.70 \\
\hline \multirow{2}{*}{$\begin{array}{l}12.3 \\
0\end{array}$} & ID & 23.0 & 47. & 0.00 & 440 & 18. & 0.40 \\
\hline & OD & 39.5 & 37. & 0.00 & 371 & 19. & 0.90 \\
\hline \multirow[b]{2}{*}{1.00} & ID & 23.7 & 45. & 0.00 & 403 & 19. & 0.40 \\
\hline & OD & 40.0 & 35. & 0.00 & 325 & 20. & 1.70 \\
\hline \multirow[b]{2}{*}{1.30} & ID & 24.5 & 43. & 0.00 & 429 & 18. & 0.40 \\
\hline & OD & 39.5 & 35. & 0.00 & 355 & 19. & 0.80 \\
\hline \multirow[b]{2}{*}{2.00} & ID & 24.5 & 45. & 0.00 & 429 & 19. & 0.40 \\
\hline & OD & 39.0 & 36. & 0.00 & 395 & 19. & 0.40 \\
\hline \multirow[b]{2}{*}{2.30} & ID & 24.4 & 47. & 0.00 & 419 & 18. & 0.40 \\
\hline & OD & 38.0 & 36. & 0.00 & 363 & 19. & 0.70 \\
\hline
\end{tabular}

\begin{tabular}{|l|l|l|l|l|l|l|l|}
\multirow{3}{*}{3.00} & ID & 23.7 & 42. & 0.00 & 443 & 18. & 0.40 \\
\cline { 2 - 9 } & OD & 38.0 & 34. & 0.00 & 355 & 19. & 0.80 \\
\hline \multirow{3}{*}{3.30} & ID & 22.6 & 43. & 0.00 & 447 & 19. & 0.40 \\
\cline { 2 - 8 } & OD & 36.5 & 37. & 0.00 & 351 & 19. & 0.60 \\
\hline \multirow{3}{*}{4.00} & ID & 22.7 & 45. & 0.00 & 437 & 18. & 0.40 \\
\cline { 2 - 8 } & OD & 35.5 & 38. & 0.00 & 342 & 19. & 0.40 \\
\hline \multirow{3}{*}{4.30} & ID & 21.9 & 47. & 0.00 & 447 & 18. & 0.40 \\
\cline { 2 - 8 } & OD & 35.0 & 38. & 0.00 & 357 & 19. & 0.90 \\
\hline \multirow{3}{*}{5.00} & ID & 21.3 & 48. & 0.00 & 467 & 18. & 0.40 \\
\cline { 2 - 8 } & OD & $\begin{array}{l}34.5 \\
0\end{array}$ & $\begin{array}{l}40 . \\
0\end{array}$ & 0.00 & 340 & 19. & 0.60 \\
& & 0 & & 6 & \\
\hline
\end{tabular}

Table 3.2 Airflow volume 2100 with without loading

\begin{tabular}{|c|c|c|c|c|c|c|c|}
\hline $\begin{array}{l}\text { Tim } \\
\mathrm{e}\end{array}$ & $\begin{array}{l}\text { Indoor } \\
\text { / }\end{array}$ & $\begin{array}{l}\text { Tem } \\
\mathrm{p}\end{array}$ & $\begin{array}{l}\mathrm{RH} \\
(\%\end{array}$ & $\begin{array}{l}\mathrm{CO} \\
(\mathrm{ppm})\end{array}$ & $\begin{array}{l}\mathrm{CO}_{2} \\
(\mathrm{ppm})\end{array}$ & $\begin{array}{l}\mathrm{O}_{2} \\
(\%\end{array}$ & $\begin{array}{l}\mathrm{RV} \\
(\mathrm{m} /\end{array}$ \\
\hline \multirow[b]{2}{*}{9.00} & ID & 29.1 & 57 & 0 & 483 & 19 & 0.4 \\
\hline & OD & 30.2 & 55 & 1 & 384 & 20 & 1.6 \\
\hline \multirow{2}{*}{9.30} & ID & 27 & 48 & 0 & 479 & 19 & 0.4 \\
\hline & OD & 32.1 & 54 & 1 & 370 & 20 & 1.2 \\
\hline \multirow[b]{2}{*}{10.0} & ID & 25.5 & 47 & 0 & 488 & 19 & 0.4 \\
\hline & OD & 34.2 & 50 & 1 & 391 & 20 & 1.4 \\
\hline \multirow{2}{*}{$\begin{array}{l}10.3 \\
0\end{array}$} & ID & 21.6 & 45 & 0 & 503 & 19 & 0.4 \\
\hline & OD & 35.6 & 50 & 1 & 371 & 20 & 1.1 \\
\hline \multirow{2}{*}{$\begin{array}{l}11.0 \\
0\end{array}$} & ID & 20.8 & 48 & 0 & 504 & 19 & 0.4 \\
\hline & OD & 37.1 & 44 & 1 & 357 & 20 & 1.3 \\
\hline \multirow{2}{*}{$\begin{array}{l}11.3 \\
0 \\
\end{array}$} & ID & 19.6 & 45 & 0 & 507 & 19 & 0.4 \\
\hline & OD & 37.4 & 43 & 0 & 364 & 20 & 1 \\
\hline \multirow{2}{*}{$\begin{array}{l}12.0 \\
0\end{array}$} & ID & 20.5 & 47 & 0 & 528 & 19 & 0.4 \\
\hline & OD & 38 & 40 & 0 & 358 & 19 & 1.2 \\
\hline \multirow{2}{*}{$\begin{array}{l}12.3 \\
0\end{array}$} & ID & 20.1 & 48 & 0 & 501 & 19 & 0.4 \\
\hline & OD & 38.2 & 39 & 0 & 351 & 20 & 0.9 \\
\hline \multirow{2}{*}{1.00} & ID & 20.9 & 45 & 0 & 511 & 19 & 0.4 \\
\hline & OD & 39.4 & 37 & 0 & 371 & 20 & 1.6 \\
\hline \multirow{2}{*}{1.30} & ID & 19.9 & 45 & 0 & 514 & 19 & 0.4 \\
\hline & OD & 39 & 37 & 0 & 335 & 20 & 1 \\
\hline \multirow{2}{*}{2.00} & ID & 19.5 & 45 & 0 & 538 & 19 & 0.4 \\
\hline & OD & 38.7 & 38 & 0 & 315 & 20 & 1.3 \\
\hline \multirow{2}{*}{2.30} & ID & 18.8 & 46 & 0 & 549 & 19 & 0.4 \\
\hline & OD & 37.5 & 40 & 0 & 352 & 20 & 1.4 \\
\hline \multirow{2}{*}{3.00} & ID & 18.7 & 46 & 0 & $\begin{array}{l}569 \\
\end{array}$ & 19 & 0.4 \\
\hline & OD & 36.5 & 40 & 0 & 376 & 20 & 1.2 \\
\hline \multirow{2}{*}{3.30} & ID & 18.8 & 49 & 0 & 561 & 19 & 0.4 \\
\hline & OD & 36.1 & 40 & 0 & 371 & 20 & 1.6 \\
\hline \multirow{2}{*}{4.00} & ID & 18.6 & 47 & 0 & 559 & 19 & 0.4 \\
\hline & OD & 35.5 & 40 & 0 & 368 & 20 & 1.5 \\
\hline \multirow{2}{*}{4.30} & ID & 18.5 & 47 & 0 & 566 & 19 & 0.4 \\
\hline & OD & 34.2 & 40 & 0 & 359 & 20 & 1.1 \\
\hline \multirow{2}{*}{5.00} & ID & 18.5 & 48 & 0 & 568 & 19 & 0.4 \\
\hline & OD & 33.4 & 42 & 0 & 348 & 20 & 0.8 \\
\hline
\end{tabular}


Table 3.3 Airflow volume 2700 with without loading

\begin{tabular}{|c|c|c|c|c|c|c|c|}
\hline $\begin{array}{l}\text { Tim } \\
\mathrm{e}\end{array}$ & $\begin{array}{l}\text { Indoor } \\
\text { / } \\
\text { Outdoo } \\
\mathrm{r}\end{array}$ & $\begin{array}{l}\text { Tem } \\
\text { p } \\
\left({ }^{\circ} \mathrm{C}\right)\end{array}$ & $\begin{array}{l}\mathrm{R} \\
\mathrm{H} \\
(\% \\
)\end{array}$ & $\begin{array}{l}\mathrm{CO} \\
\text { (ppm } \\
\text { ) }\end{array}$ & $\begin{array}{l}\mathrm{CO}_{2} \\
\text { (ppm } \\
\text { ) }\end{array}$ & $\begin{array}{l}\mathrm{O}_{2} \\
(\% \\
)\end{array}$ & $\begin{array}{l}\mathrm{RV} \\
(\mathrm{m} / \mathrm{s} \\
)\end{array}$ \\
\hline \multirow{2}{*}{9.00} & ID & 29.8 & 56 & 0 & 430 & 20 & \begin{tabular}{|l|l|}
0.4 \\
\end{tabular} \\
\hline & OD & 30.5 & 55 & 1 & 381 & 20 & \begin{tabular}{|l}
1.6 \\
\end{tabular} \\
\hline \multirow{2}{*}{9.30} & ID & 26.6 & 49 & 0 & 426 & 19 & \begin{tabular}{|l|}
0.4 \\
\end{tabular} \\
\hline & OD & 32.3 & 54 & 1 & 367 & 20 & \begin{tabular}{|l|}
1.9 \\
\end{tabular} \\
\hline \multirow{2}{*}{$\begin{array}{l}10.0 \\
0\end{array}$} & ID & 25.2 & 48 & 0 & 429 & 19 & 0.4 \\
\hline & OD & 34.2 & 50 & 1 & 397 & 20 & 1.3 \\
\hline \multirow{2}{*}{$\begin{array}{l}10.3 \\
0\end{array}$} & ID & 21.4 & 45 & 0 & 457 & 19 & \begin{tabular}{|l|}
0.4 \\
\end{tabular} \\
\hline & OD & 35.2 & 49 & 1 & 371 & 20 & $\mid 1.3$ \\
\hline \multirow{2}{*}{$\begin{array}{l}11.0 \\
0\end{array}$} & ID & 20.3 & 48 & 0 & 449 & 19 & 0.4 \\
\hline & OD & 36.5 & 45 & 0 & 357 & 20 & \begin{tabular}{|l|}
1.1 \\
\end{tabular} \\
\hline \multirow{2}{*}{$\begin{array}{l}11.3 \\
0\end{array}$} & ID & 19.8 & 51 & 0 & 455 & 19 & \begin{tabular}{|l|}
0.4 \\
\end{tabular} \\
\hline & OD & 37 & 43 & 0 & 364 & 20 & \begin{tabular}{|l|}
0.7 \\
\end{tabular} \\
\hline \multirow{2}{*}{$\begin{array}{l}12.0 \\
0\end{array}$} & ID & 19.8 & 50 & 0 & 455 & 19 & 0.4 \\
\hline & OD & 37.5 & 41 & 0 & 367 & 21 & \begin{tabular}{|l|}
1.4 \\
\end{tabular} \\
\hline \multirow{2}{*}{$\begin{array}{l}12.3 \\
0\end{array}$} & ID & 20.2 & 49 & 0 & 465 & 19 & 0.4 \\
\hline & OD & 37.1 & 40 & 0 & 353 & 20 & 0.6 \\
\hline \multirow{2}{*}{1.00} & ID & 20.8 & 49 & 0 & 447 & 19 & \begin{tabular}{|l|} 
\\
\end{tabular} \\
\hline & OD & 37.9 & 37 & 0 & 377 & 20 & \begin{tabular}{|l|}
0.8 \\
\end{tabular} \\
\hline \multirow{2}{*}{1.30} & ID & 21.1 & 48 & 0 & 473 & 19 & \begin{tabular}{|l|l}
0.4 \\
\end{tabular} \\
\hline & OD & 38.5 & 40 & 0 & 389 & 20 & \begin{tabular}{|l|}
1.7 \\
\end{tabular} \\
\hline \multirow{2}{*}{2.00} & ID & 21.5 & 47 & 0 & 477 & 19 & 0.4 \\
\hline & OD & 39.3 & 40 & 0 & 371 & 20 & \begin{tabular}{|l|l}
0.9 \\
\end{tabular} \\
\hline \multirow{2}{*}{2.30} & ID & 21.1 & 47 & 0 & 498 & 19 & \begin{tabular}{|l}
0.4 \\
\end{tabular} \\
\hline & OD & 38.2 & 42 & 0 & 383 & 20 & 0.8 \\
\hline \multirow{2}{*}{3.00} & ID & 19.8 & 48 & 0 & 511 & 19 & \begin{tabular}{|l|}
0.4 \\
\end{tabular} \\
\hline & OD & 37.3 & 40 & 0 & 371 & 20 & \begin{tabular}{|l}
1.2 \\
\end{tabular} \\
\hline \multirow{2}{*}{3.30} & ID & 19.2 & 49 & 0 & 487 & 19 & 0.4 \\
\hline & OD & 36 & 43 & 0 & 383 & 20 & \begin{tabular}{|l|}
1.1 \\
\end{tabular} \\
\hline \multirow{2}{*}{4.00} & ID & 18.8 & 49 & 0 & 521 & 19 & 0.4 \\
\hline & OD & 35 & 44 & 0 & 405 & 20 & \begin{tabular}{|l|}
1.6 \\
\end{tabular} \\
\hline \multirow{2}{*}{4.30} & ID & 19.4 & 50 & 0 & 558 & 19 & 0.4 \\
\hline & OD & 34.3 & 44 & 0 & 402 & 21 & \begin{tabular}{|l|}
2.1 \\
\end{tabular} \\
\hline \multirow{2}{*}{5.00} & ID & 18.5 & 52 & 0 & 553 & 19 & \begin{tabular}{|l|l} 
\\
\end{tabular} \\
\hline & OD & 33.8 & 46 & 0 & 393 & 20 & \begin{tabular}{|l}
1.9 \\
\end{tabular} \\
\hline
\end{tabular}

The measured variables of without load in meeting hall are tabulated. Readings are identified better comfort zone with efficient energy consumption. From the above tables without load the meeting hall obtained thermal comfort below $1400 \mathrm{cfm}$ as per ASHARE standard. Without human load the meeting hall no need to too cool.
Table 3.4 Airflow volume 1400 with partial load

\begin{tabular}{|c|c|c|c|c|c|c|c|}
\hline $\begin{array}{l}\text { Tim } \\
\mathrm{e}\end{array}$ & $\begin{array}{l}\text { Indoor } \\
\text { / } \\
\text { Outdoo } \\
\mathrm{r}\end{array}$ & $\begin{array}{l}\text { Tem } \\
\text { p } \\
\left({ }^{\circ} \mathrm{C}\right)\end{array}$ & $\begin{array}{l}\mathrm{R} \\
\mathrm{H} \\
(\% \\
)\end{array}$ & $\begin{array}{l}\mathrm{CO} \\
\text { (ppm } \\
\text { ) }\end{array}$ & $\begin{array}{l}\mathrm{CO}_{2} \\
\text { (ppm } \\
\text { ) }\end{array}$ & $\begin{array}{l}\mathrm{O}_{2} \\
(\% \\
)\end{array}$ & $\begin{array}{l}\mathrm{RV} \\
(\mathrm{m} / \mathrm{s} \\
)\end{array}$ \\
\hline \multirow{2}{*}{9.00} & ID & 30 & 56 & 0 & 429 & 20 & 0.4 \\
\hline & OD & 31.5 & 54 & 2 & 387 & 20 & 1.7 \\
\hline \multirow{2}{*}{9.30} & ID & 27.2 & 52 & 0 & 419 & 19 & 0.4 \\
\hline & OD & 33 & 53 & 2 & 371 & 20 & 1.1 \\
\hline \multirow{2}{*}{$\begin{array}{l}10.0 \\
0\end{array}$} & ID & 26 & 51 & 0 & 431 & 19 & 0.4 \\
\hline & OD & 35 & 50 & 2 & 393 & 20 & 1.3 \\
\hline \multirow{2}{*}{$\begin{array}{l}10.3 \\
0\end{array}$} & ID & 24 & 50 & 0 & 413 & 19 & 0.4 \\
\hline & OD & 35.5 & 50 & 1 & 374 & 20 & 1 \\
\hline \multirow{2}{*}{$\begin{array}{l}11.0 \\
0\end{array}$} & ID & 24 & 50 & 0 & 422 & 19 & 0.4 \\
\hline & OD & 37 & 44 & 1 & 351 & 21 & 1.2 \\
\hline \multirow{2}{*}{$\begin{array}{l}11.3 \\
0\end{array}$} & ID & 23.3 & 52 & 0 & 411 & 19 & 0.4 \\
\hline & OD & 37.2 & 43 & 0 & 361 & 20 & 0.9 \\
\hline \multirow{2}{*}{$\begin{array}{l}12.0 \\
0\end{array}$} & ID & 23.2 & 46 & 0 & 431 & 19 & 0.4 \\
\hline & OD & 38 & 41 & 0 & 362 & 20 & 1.2 \\
\hline \multirow{2}{*}{$\begin{array}{l}12.3 \\
0\end{array}$} & ID & 22.7 & 47 & 0 & 439 & 19 & 0.4 \\
\hline & OD & 37.8 & 39 & 0 & 359 & 20 & 1.5 \\
\hline \multirow{2}{*}{1.00} & ID & 23.6 & 46 & 0 & 441 & 19 & 0.4 \\
\hline & OD & 38 & 37 & 0 & 373 & 20 & 2.2 \\
\hline \multirow{2}{*}{1.30} & ID & 22.8 & 44 & 0 & 407 & 19 & 0.4 \\
\hline & OD & 37.4 & 40 & 0 & 315 & 20 & 0.9 \\
\hline \multirow{2}{*}{2.00} & ID & 22.1 & 44 & 0 & 392 & 19 & 0.4 \\
\hline & OD & 37 & 44 & 0 & 265 & 19 & 1.1 \\
\hline \multirow{2}{*}{2.30} & ID & 22.4 & 45 & 0 & 439 & 19 & 0.4 \\
\hline & OD & 37.1 & 44 & 0 & 347 & 20 & 1.4 \\
\hline \multirow{2}{*}{3.00} & ID & 21.8 & 47 & 0 & 437 & 19 & 0.4 \\
\hline & OD & 37 & 40 & 0 & 372 & 20 & 1.1 \\
\hline \multirow{2}{*}{3.30} & ID & 22.6 & 45 & 0 & 443 & 19 & 0.4 \\
\hline & OD & 36.2 & 42 & 40 & 371 & 20 & 1.7 \\
\hline \multirow{2}{*}{4.00} & ID & 21.9 & 48 & 0 & 529 & 19 & 0.4 \\
\hline & OD & 35.5 & 44 & 0 & 365 & 19 & 1.5 \\
\hline \multirow{2}{*}{4.30} & ID & 22.2 & 49 & 0 & 563 & 19 & 0.4 \\
\hline & OD & 35.1 & 41 & 0 & 353 & 20 & 1.1 \\
\hline \multirow{2}{*}{5.00} & ID & 21.6 & 51 & 0 & 597 & 19 & 0.4 \\
\hline & OD & 34.5 & 40 & 0 & 345 & 19 & 0.6 \\
\hline
\end{tabular}

Table 3.5 Airflow volume 2100 with partial load

\begin{tabular}{|c|c|c|c|c|c|c|c|}
\hline $\begin{array}{l}\text { Tim } \\
\mathrm{e}\end{array}$ & $\begin{array}{l}\text { Indoor } \\
/ \\
\text { Outdoo } \\
\text { r }\end{array}$ & $\begin{array}{l}\text { Tem } \\
\mathrm{p} \\
\left({ }^{\circ} \mathrm{C}\right)\end{array}$ & $\begin{array}{l}\mathrm{R} \\
\mathrm{H} \\
(\% \\
)\end{array}$ & $\begin{array}{l}\mathrm{CO} \\
\text { (ppm } \\
\text { ) }\end{array}$ & $\begin{array}{l}\mathrm{CO}_{2} \\
\text { (ppm } \\
\text { ) }\end{array}$ & $\begin{array}{l}\mathrm{O}_{2} \\
(\% \\
)\end{array}$ & $\begin{array}{l}\mathrm{RV} \\
(\mathrm{m} / \mathrm{s} \\
)\end{array}$ \\
\hline \multirow{2}{*}{9.00} & ID & 29 & 56 & 0 & 489 & 19 & 0.4 \\
\hline & OD & 29.8 & 56 & 0 & 417 & 20 & 0.9 \\
\hline 9.30 & ID & 27.3 & 47 & 0 & 513 & 19 & 0.4 \\
\hline
\end{tabular}




\begin{tabular}{|c|c|c|c|c|c|c|c|}
\hline & OD & 30.5 & 52 & 0 & 409 & 19 & 0.5 \\
\hline \multirow{2}{*}{$\begin{array}{l}10.0 \\
0\end{array}$} & ID & 24.5 & 47 & 0 & 525 & 19 & 0.4 \\
\hline & OD & 31 & 50 & 0 & 421 & 20 & 0.7 \\
\hline \multirow{2}{*}{$\begin{array}{l}10.3 \\
0\end{array}$} & ID & 23.3 & 47 & 0 & 537 & 19 & 0.4 \\
\hline & OD & 31.1 & 47 & 0 & 416 & 20 & 0.8 \\
\hline \multirow{2}{*}{$\begin{array}{l}11.0 \\
0\end{array}$} & ID & 22.6 & 45 & 0 & $\begin{array}{l}538 \\
\end{array}$ & 19 & 0.4 \\
\hline & OD & 31.3 & 45 & 0 & 399 & 19 & 0.5 \\
\hline \multirow{2}{*}{$\begin{array}{l}11.3 \\
0\end{array}$} & ID & 22.8 & 45 & 0 & 549 & 19 & 0.4 \\
\hline & OD & 32.7 & 40 & 0 & 371 & 20 & 0.8 \\
\hline \multirow{2}{*}{$\begin{array}{l}12.0 \\
0\end{array}$} & ID & 23.7 & 45 & 0 & 559 & 19 & 0.4 \\
\hline & OD & 34 & 38 & 0 & 359 & 20 & 0.7 \\
\hline \multirow{2}{*}{$\begin{array}{l}12.3 \\
0\end{array}$} & ID & 25.1 & 41 & 0 & 549 & 19 & 0.4 \\
\hline & OD & 36.1 & 38 & 0 & 372 & 20 & 1 \\
\hline \multirow{2}{*}{1.00} & ID & 23.3 & 42 & 0 & 554 & 19 & 0.4 \\
\hline & OD & 38.4 & 36 & 0 & 362 & 20 & 0.9 \\
\hline \multirow{2}{*}{1.30} & ID & 22.2 & 45 & 0 & 569 & 19 & 0.4 \\
\hline & OD & 38.5 & 35 & 0 & 361 & 20 & 1.1 \\
\hline \multirow{2}{*}{2.00} & ID & 22.1 & 45 & 0 & 581 & 19 & 0.4 \\
\hline & OD & 39.1 & 32 & 0 & 355 & 20 & 0.6 \\
\hline \multirow{2}{*}{2.30} & ID & 21.4 & 45 & 0 & 582 & 19 & 0.4 \\
\hline & OD & 39 & 32 & 0 & 347 & 20 & 0.5 \\
\hline \multirow{2}{*}{3.00} & ID & 21 & 44 & 0 & \begin{tabular}{|l|l|}
588 \\
\end{tabular} & 19 & 0.4 \\
\hline & OD & 37.3 & 38 & 0 & 367 & 20 & 0.7 \\
\hline \multirow{2}{*}{3.30} & ID & 20.7 & 44 & 0 & 591 & 19 & 0.4 \\
\hline & OD & 36.1 & 39 & 0 & 369 & 20 & 0.8 \\
\hline \multirow{2}{*}{4.00} & ID & 19.2 & 44 & 0 & 609 & 19 & 0.4 \\
\hline & OD & 35 & 41 & 0 & 371 & 20 & 1 \\
\hline \multirow{2}{*}{4.30} & ID & 18.5 & 46 & 0 & 605 & 19 & 0.4 \\
\hline & OD & 33.9 & 44 & 0 & 391 & 19 & 1 \\
\hline \multirow{2}{*}{5.00} & ID & 18.5 & 46 & 0 & 632 & 19 & 0.4 \\
\hline & OD & 31.2 & 46 & 0 & 402 & 19 & 0.6 \\
\hline
\end{tabular}

\begin{tabular}{|l|l|l|l|l|l|l|l|}
0 & OD & 31 & 50 & 0 & 347 & 20 & 2.4 \\
\hline \multirow{2}{*}{$\begin{array}{l}12.3 \\
0\end{array}$} & ID & 20.8 & 49 & 0 & 523 & 19 & 0.4 \\
\cline { 2 - 8 } & OD & 31.1 & 50 & 0 & 325 & 20 & 2.5 \\
\hline \multirow{3}{*}{1.00} & ID & 19.3 & 49 & 0 & 534 & 19 & 0.4 \\
\cline { 2 - 8 } & OD & 32 & 50 & 0 & 302 & 20 & 2.7 \\
\hline \multirow{3}{*}{1.30} & ID & 18.9 & 48 & 0 & 517 & 19 & 0.4 \\
\cline { 2 - 8 } & OD & 31.7 & 50 & 0 & 351 & 20 & 2.3 \\
\hline \multirow{3}{*}{2.00} & ID & 18.4 & 46 & 0 & 533 & 19 & 0.4 \\
\cline { 2 - 8 } & OD & 32 & 49 & 0 & 342 & 20 & 2.8 \\
\hline \multirow{3}{*}{2.30} & ID & 18.8 & 52 & 0 & 554 & 19 & 0.4 \\
\cline { 2 - 8 } & OD & 32.6 & 47 & 0 & 367 & 19 & 2.9 \\
\hline \multirow{3}{*}{3.00} & ID & 18.7 & 45 & 0 & 606 & 19 & 0.4 \\
\cline { 2 - 8 } & OD & 33.5 & 45 & 0 & 380 & 19 & 2.7 \\
\hline \multirow{2}{*}{3.30} & ID & 18.5 & 45 & 0 & 613 & 19 & 0.4 \\
\cline { 2 - 8 } & OD & 33.1 & 44 & 0 & 373 & 20 & 2.8 \\
\hline \multirow{2}{*}{4.00} & ID & 18.7 & 45 & 0 & 577 & 19 & 0.4 \\
\cline { 2 - 8 } & OD & 33 & 42 & 0 & 366 & 20 & 3.2 \\
\hline \multirow{2}{*}{4.30} & ID & 18.4 & 47 & 0 & 617 & 19 & 0.4 \\
\cline { 2 - 8 } & OD & 32.1 & 40 & 0 & 361 & 20 & 3.1 \\
\hline \multirow{2}{*}{5.00} & ID & 18.3 & 47 & 0 & 621 & 19 & 0.4 \\
\cline { 2 - 7 } & OD & 31.5 & 40 & 0 & 355 & 20 & 3.3 \\
\hline
\end{tabular}

The measured variables of with partial load in meeting hall are tabulated. Here the partial load means only consider furniture and indoor equipments. Readings are identified better comfort zone with efficient energy consumption. From the above tables without load the meeting hall obtained thermal comfort below $1400 \mathrm{cfm}$ as per ASHARE standard temperature level. Without human load the meeting hall no need to too cool.

Table 3.7 CFM: 1400 -FULL LOAD

Table 3.6 Airflow volume 2700 with partial load

\begin{tabular}{|l|l|l|l|l|l|l|l|}
\hline \multirow{2}{*}{$\begin{array}{l}\text { Tim } \\
\mathrm{e}\end{array}$} & $\begin{array}{l}\text { Indoor } \\
\text { I }\end{array}$ & $\begin{array}{l}\mathrm{Tem} \\
\mathrm{p} \\
\mathrm{r}\end{array}$ & $\begin{array}{l}\mathrm{R} \\
\left({ }^{\circ} \mathrm{C}\right)\end{array}$ & $\begin{array}{l}\mathrm{CO} \\
(\% \\
(\mathrm{ppm}\end{array}$ & $\begin{array}{l}\mathrm{CO}_{2} \\
(\mathrm{ppm}\end{array}$ & $\begin{array}{l}\mathrm{O}_{2} \\
(\%\end{array}$ & $\begin{array}{l}\mathrm{RV} \\
(\mathrm{m} / \mathrm{s}\end{array}$ \\
\hline \multirow{2}{*}{9.00} & $\mathrm{ID}$ & 29 & 57 & 0 & 442 & 19 & 0.4 \\
\cline { 2 - 9 } & OD & 29.5 & 56 & 0 & 382 & 20 & 2.4 \\
\hline \multirow{2}{*}{9.30} & $\mathrm{ID}$ & 26.9 & 47 & 0 & 459 & 19 & 0.4 \\
\cline { 2 - 9 } & OD & 30 & 54 & 0 & 372 & 20 & 2.7 \\
\hline 10.0 & $\mathrm{ID}$ & 25.5 & 47 & 0 & 464 & 19 & 0.4 \\
\cline { 2 - 9 } 0 & OD & 30.4 & 54 & 0 & 379 & 19 & 2.5 \\
\hline 10.3 & $\mathrm{ID}$ & 23.8 & 47 & 0 & 459 & 19 & 0.4 \\
\cline { 2 - 8 } 0 & OD & 29.2 & 56 & 0 & 384 & 20 & 2.4 \\
\hline 11.0 & $\mathrm{ID}$ & 22.4 & 48 & 0 & 445 & 19 & 0.4 \\
\cline { 2 - 8 } 0 & OD & 28.9 & 57 & 0 & 376 & 20 & 3.1 \\
\hline 11.3 & $\mathrm{ID}$ & 22.8 & 47 & 0 & 459 & 19 & 0.4 \\
\cline { 2 - 8 } 0 & OD & 30.5 & 50 & 0 & 351 & 20 & 2.7 \\
\hline 12.0 & $\mathrm{ID}$ & 22.1 & 48 & 0 & 493 & 19 & 0.4 \\
\hline
\end{tabular}

\begin{tabular}{|c|c|c|c|c|c|c|c|}
\hline $\begin{array}{l}\text { Tim } \\
\mathrm{e}\end{array}$ & $\begin{array}{l}\text { Indoor } \\
\text { / } \\
\text { Outdoo } \\
\mathrm{r}\end{array}$ & $\begin{array}{l}\text { Tem } \\
\mathrm{p} \\
\left({ }^{\circ} \mathrm{C}\right)\end{array}$ & $\begin{array}{l}\mathrm{R} \\
\mathrm{H} \\
(\% \\
)\end{array}$ & $\begin{array}{l}\mathrm{CO} \\
\text { (ppm } \\
\text { ) }\end{array}$ & $\begin{array}{l}\mathrm{CO}_{2} \\
\text { (ppm } \\
\text { ) }\end{array}$ & $\begin{array}{l}\mathrm{O}_{2} \\
(\% \\
)\end{array}$ & $\begin{array}{l}\mathrm{RV} \\
(\mathrm{m} / \mathrm{s} \\
)\end{array}$ \\
\hline \multirow{2}{*}{9.00} & ID & 29.2 & 56 & 0 & 417 & 20 & 0.4 \\
\hline & OD & 30.1 & 55 & 1 & 357 & 20 & 2.2 \\
\hline \multirow{2}{*}{9.30} & ID & 29 & 52 & 0 & 1249 & 19 & 0.4 \\
\hline & OD & 31.5 & 54 & 1 & 379 & 20 & 2.4 \\
\hline \multirow{2}{*}{$\begin{array}{l}10.0 \\
0\end{array}$} & ID & 28.2 & 49 & 0 & 1725 & 19 & 0.4 \\
\hline & OD & 33 & 52 & 1 & 384 & 20 & 2.8 \\
\hline \multirow{2}{*}{$\begin{array}{l}10.3 \\
0\end{array}$} & ID & 28 & 49 & 0 & 2497 & 19 & 0.4 \\
\hline & OD & 34.5 & 49 & 1 & 375 & 20 & 3 \\
\hline \multirow{2}{*}{$\begin{array}{l}11.0 \\
0\end{array}$} & ID & 27.6 & 48 & 0 & 3011 & 19 & 0.4 \\
\hline & OD & 36.1 & 48 & 0 & 391 & 20 & 2.4 \\
\hline \multirow{2}{*}{$\begin{array}{l}11.3 \\
0\end{array}$} & ID & 27.3 & 47 & 0 & 3469 & 19 & 0.4 \\
\hline & OD & 37.3 & 45 & 0 & 361 & 20 & 2.2 \\
\hline \multirow{2}{*}{$\begin{array}{l}12.0 \\
0\end{array}$} & ID & 26.8 & 47 & 0 & 3664 & 19 & 0.4 \\
\hline & OD & 37.9 & 42 & 0 & 374 & 20 & 2.3 \\
\hline \multirow{2}{*}{$\begin{array}{l}12.3 \\
0\end{array}$} & ID & 27.1 & 46 & 0 & 3754 & 19 & 0.4 \\
\hline & OD & 38.4 & 40 & 0 & 359 & 20 & 2.7 \\
\hline
\end{tabular}




\begin{tabular}{|l|l|l|l|l|l|l|l|}
\multirow{2}{*}{1.00} & ID & 26.8 & 46 & 0 & 3939 & 19 & 0.4 \\
\cline { 2 - 8 } & OD & 39.8 & 36 & 0 & 354 & 20 & 3.1 \\
\hline \multirow{2}{*}{1.30} & ID & 26.5 & 46 & 0 & 4097 & 19 & 0.4 \\
\cline { 2 - 8 } & OD & 40.4 & 34 & 0 & 341 & 20 & 1.9 \\
\hline \multirow{2}{*}{2.00} & ID & 26.5 & 46 & 0 & 4157 & 19 & 0.4 \\
\cline { 2 - 8 } & OD & 41 & 31 & 0 & 347 & 20 & 2.6 \\
\hline \multirow{2}{*}{2.30} & ID & 26.4 & 47 & 0 & 4167 & 19 & 0.4 \\
\cline { 2 - 8 } & OD & 40.1 & 33 & 0 & 371 & 20 & 2.8 \\
\hline \multirow{2}{*}{3.00} & ID & 26.5 & 47 & 0 & 4159 & 19 & 0.4 \\
\cline { 2 - 8 } & OD & 39 & 36 & 0 & 364 & 20 & 2.1 \\
\hline \multirow{2}{*}{3.30} & ID & 26.2 & 48 & 0 & 4198 & 19 & 0.4 \\
\cline { 2 - 8 } & OD & 37.4 & 37 & 0 & 383 & 20 & 2.3 \\
\hline \multirow{2}{*}{4.00} & ID & 26.1 & 48 & 0 & 4191 & 19 & 0.4 \\
\cline { 2 - 8 } & OD & 36.2 & 39 & 0 & 379 & 20 & 2 \\
\hline \multirow{2}{*}{4.30} & ID & 26.2 & 49 & 0 & 4207 & 19 & 0.4 \\
\cline { 2 - 8 } & OD & 34.3 & 43 & 0 & 392 & 20 & 1.8 \\
\hline \multirow{2}{*}{5.00} & ID & 26 & 49 & 0 & 4209 & 19 & 0.4 \\
\cline { 2 - 7 } & OD & 32.1 & 47 & 0 & 384 & 20 & 2 \\
\hline
\end{tabular}

\begin{tabular}{|l|l|l|l|l|l|l|l|}
\multirow{2}{*}{3.30} & ID & 24.8 & 47 & 0 & 4339 & 19 & 0.4 \\
\cline { 2 - 8 } & OD & 38.4 & 37 & 0 & 371 & 20 & 2.7 \\
\hline \multirow{2}{*}{4.00} & ID & 24.7 & 47 & 0 & 4343 & 19 & 0.4 \\
\cline { 2 - 8 } & OD & 37.1 & 38 & 0 & 383 & 20 & 2.4 \\
\hline \multirow{2}{*}{4.30} & ID & 24.7 & 48 & 0 & 4339 & 19 & 0.4 \\
\cline { 2 - 8 } & OD & 35.2 & 44 & 0 & 357 & 20 & 3 \\
\hline \multirow{2}{*}{5.00} & ID & 24.5 & 49 & 0 & 4347 & 19 & 0.4 \\
\cline { 2 - 8 } & OD & 33.1 & 48 & 0 & 394 & 20 & 2.8 \\
\hline
\end{tabular}

Table 3.8 CFM: 2100-FULL LOAD

\begin{tabular}{|c|c|c|c|c|c|c|c|}
\hline $\begin{array}{l}\text { Tim } \\
\mathrm{e}\end{array}$ & $\begin{array}{l}\text { Indoor } \\
\text { / } \\
\text { Outdoo } \\
\mathrm{r}\end{array}$ & $\begin{array}{l}\text { Tem } \\
\mathrm{p} \\
\left({ }^{\circ} \mathrm{C}\right)\end{array}$ & $\begin{array}{l}\mathrm{R} \\
\mathrm{H} \\
(\% \\
)\end{array}$ & $\begin{array}{l}\mathrm{CO} \\
\text { (ppm } \\
\text { ) }\end{array}$ & $\begin{array}{l}\mathrm{CO}_{2} \\
\text { (ppm } \\
\text { ) }\end{array}$ & $\begin{array}{l}\mathrm{O}_{2} \\
(\% \\
)\end{array}$ & $\begin{array}{l}\mathrm{RV} \\
(\mathrm{m} / \mathrm{s} \\
)\end{array}$ \\
\hline \multirow{2}{*}{9.00} & ID & 28.3 & 53 & 0 & 443 & 19 & 0.4 \\
\hline & OD & 29.5 & 56 & 1 & 371 & 20 & 1.8 \\
\hline \multirow{2}{*}{9.30} & ID & 27.4 & 52 & 0 & 1339 & 19 & 0.4 \\
\hline & OD & 31.1 & 54 & 1 & 367 & 20 & 2.3 \\
\hline \multirow{2}{*}{$\begin{array}{l}10.0 \\
0\end{array}$} & ID & 26.5 & 50 & 0 & 1795 & 19 & 0.4 \\
\hline & OD & 33.4 & 52 & 1 & 384 & 20 & 2.5 \\
\hline \multirow{2}{*}{$\begin{array}{l}10.3 \\
0 \\
\end{array}$} & ID & 25.7 & 49 & 0 & 2361 & 19 & 0.4 \\
\hline & OD & 34.8 & 49 & 0 & 393 & 20 & 2.6 \\
\hline \multirow{2}{*}{$\begin{array}{l}11.0 \\
0\end{array}$} & ID & 25.5 & 49 & 0 & 2815 & 19 & 0.4 \\
\hline & OD & 35.7 & 46 & 0 & 368 & 20 & 1.9 \\
\hline \multirow{2}{*}{$\begin{array}{l}11.3 \\
0\end{array}$} & ID & 24.9 & 48 & 0 & 3505 & 19 & 0.4 \\
\hline & OD & 37 & 43 & 0 & 371 & 20 & 2.2 \\
\hline \multirow{2}{*}{$\begin{array}{l}12.0 \\
0 \\
\end{array}$} & ID & 25.5 & 48 & 0 & 3689 & 19 & 0.4 \\
\hline & OD & 37.7 & 42 & 0 & 353 & 20 & 2.1 \\
\hline \multirow{2}{*}{$\begin{array}{l}12.3 \\
0\end{array}$} & ID & 25.4 & 47 & 0 & 3869 & 19 & 0.4 \\
\hline & OD & 38.1 & 40 & 0 & 348 & 20 & 2.1 \\
\hline \multirow{2}{*}{1.00} & ID & 25.2 & 45 & 0 & 4092 & 19 & 0.4 \\
\hline & OD & 38.5 & 36 & 0 & 361 & 20 & 1.9 \\
\hline \multirow{2}{*}{1.30} & ID & 25.2 & 46 & 0 & 4227 & 19 & 0.4 \\
\hline & OD & 39.2 & 35 & 0 & 338 & 20 & 2.5 \\
\hline \multirow{2}{*}{2.00} & ID & 25.2 & 46 & 0 & 4294 & 19 & 0.4 \\
\hline & OD & 39.5 & 35 & 0 & 345 & 20 & 2.4 \\
\hline \multirow{2}{*}{2.30} & ID & 25 & 47 & 0 & 4318 & 19 & 0.4 \\
\hline & OD & 40 & 33 & 0 & 357 & 20 & 2.7 \\
\hline \multirow{2}{*}{3.00} & ID & 24.9 & 47 & 0 & 4318 & 19 & 0.4 \\
\hline & OD & 39.1 & 35 & 0 & 367 & 20 & 2.3 \\
\hline
\end{tabular}

Table 3.9 CFM: 2700-FULL LOAD

\begin{tabular}{|c|c|c|c|c|c|c|c|}
\hline Time & $\begin{array}{l}\text { Indoor / } \\
\text { Outdoor }\end{array}$ & $\begin{array}{l}\text { Temp } \\
\left({ }^{\circ} \mathrm{C}\right)\end{array}$ & $\begin{array}{l}\mathrm{RH} \\
(\%)\end{array}$ & $\begin{array}{l}\mathrm{CO} \\
(\mathrm{ppm})\end{array}$ & $\begin{array}{l}\mathrm{CO}_{2} \\
(\mathrm{ppm})\end{array}$ & $\begin{array}{l}\mathrm{O}_{2} \\
(\%)\end{array}$ & $\begin{array}{l}\mathrm{RV} \\
(\mathrm{m} / \mathrm{s})\end{array}$ \\
\hline \multirow[t]{2}{*}{9.00} & ID & 28.5 & 54 & 0 & 464 & 19 & 0.4 \\
\hline & OD & 30.2 & 55 & 1 & 392 & 20 & 1.8 \\
\hline \multirow[t]{2}{*}{9.30} & ID & 26.8 & 50 & 0 & 1287 & 19 & 0.4 \\
\hline & OD & 32.3 & 53 & 1 & 378 & 20 & 1.2 \\
\hline \multirow[t]{2}{*}{10.00} & ID & 25.9 & 49 & 0 & 1769 & 19 & 0.4 \\
\hline & OD & 34.7 & 50 & 1 & 398 & 20 & 1.6 \\
\hline \multirow[t]{2}{*}{10.30} & ID & 24.9 & 49 & 0 & 2472 & 19 & 0.4 \\
\hline & OD & 35.8 & 50 & 1 & 377 & 20 & 1.1 \\
\hline \multirow[t]{2}{*}{11.00} & ID & 24.4 & 50 & 0 & 2943 & 19 & 0.4 \\
\hline & OD & 37.1 & 44 & 1 & 362 & 20 & 1.4 \\
\hline \multirow[t]{2}{*}{11.30} & ID & 25 & 48 & 0 & 3682 & 19 & 0.4 \\
\hline & OD & 37.5 & 43 & 0 & 357 & 20 & 1.1 \\
\hline \multirow[t]{2}{*}{12.00} & ID & 24.5 & 47 & 0 & 3861 & 19 & 0.4 \\
\hline & OD & 38 & 41 & 0 & 367 & 20 & 1.4 \\
\hline \multirow[t]{2}{*}{12.30} & ID & 24.6 & 47 & 0 & 4109 & 19 & 0.4 \\
\hline & OD & 38.4 & 39 & 0 & 352 & 20 & 1.9 \\
\hline \multirow[t]{2}{*}{1.00} & ID & 25.1 & 47 & 0 & 4287 & $\begin{array}{l}19 \\
\end{array}$ & 0.4 \\
\hline & OD & 39.5 & 37 & 0 & 381 & 20 & 2 \\
\hline \multirow[t]{2}{*}{1.30} & ID & 24.4 & 46 & 0 & 4410 & 19 & 0.4 \\
\hline & OD & 40.2 & 34 & 0 & 357 & 20 & 1.2 \\
\hline \multirow[t]{2}{*}{2.00} & ID & 24.4 & 46 & 0 & 4472 & 19 & 0.4 \\
\hline & OD & 40 & 36 & 0 & 332 & 20 & 1.3 \\
\hline \multirow[t]{2}{*}{2.30} & ID & 24.1 & 46 & 0 & 4491 & 19 & 0.4 \\
\hline & OD & 39.1 & 38 & 0 & 364 & 19 & 1.1 \\
\hline \multirow[t]{2}{*}{3.00} & ID & 24.1 & 46 & 0 & 4519 & 19 & 0.4 \\
\hline & OD & 38 & 40 & 0 & 365 & 20 & 1.7 \\
\hline \multirow[t]{2}{*}{3.30} & ID & 24.1 & 46 & 0 & 4532 & 19 & 0.4 \\
\hline & OD & 37.2 & 41 & 0 & 374 & 20 & 1.1 \\
\hline \multirow[t]{2}{*}{4.00} & ID & 24.1 & 47 & 0 & 4537 & 19 & 0.4 \\
\hline & OD & 35.4 & 43 & 0 & 359 & 20 & 1.5 \\
\hline \multirow[t]{2}{*}{4.30} & ID & 23.9 & 47 & 0 & 4547 & 19 & 0.4 \\
\hline & OD & 34.1 & 46 & 0 & 384 & 19 & 1.6 \\
\hline \multirow{2}{*}{5.00} & ID & 23.9 & 47 & 0 & 4559 & 19 & 0.4 \\
\hline & OD & 32.9 & 48 & 0 & 394 & 20 & 1.2 \\
\hline
\end{tabular}

The measured variables of with Full load in meeting hall are tabulated. Here the full load means only consider furniture, indoor equipments and human load. Readings are identified 
better comfort zone with efficient energy consumption. From the above tables without load the meeting hall obtained thermal comfort below $2100 \mathrm{cfm}$ as per ASHARE standard temperature level.

Table.3.6,3.7,3.8.Corbon dioxide is constantly increased in the room and it is minimum at morning 9.30am and maximum at evening $5.00 \mathrm{pm}$. Recommended ASHRAE standard $\mathrm{CO}_{2}$ value for buildings in indoor is $1000 \mathrm{ppm}$. The Experimental result $\mathrm{CO}_{2}$ is crossing maximum value as per ASHARE in meeting hall with occupants of 45 peoples in $1400 \mathrm{cfm}, 2100 \mathrm{cfm}$, and $2700 \mathrm{cfm}$. Empty and Partial load is taken because of reference purposes.

Table.3.6,3.7,3.8. When the air conditioner is switch on at $9.00 \mathrm{am}$ and inner temperature of the room is decreased slowly. In afternoon time period the $12.30 \mathrm{pm}$ to $2.30 \mathrm{pm}$ the inner room temperature is slightly increase because of outdoor temperature was increased and after $3.00 \mathrm{pm}$ the indoor air temperature is decreased. Recommended ASHRAE standard summer temperature value for buildings in indoor is $22.8-26.1{ }^{\circ} \mathrm{C}^{[2]}$ in India. The experimental result obtained as per standard in the flow of $2100 \mathrm{cfm}$ and $2700 \mathrm{cfm}$.

Table.3.6,3.7,3.8. When the air conditioner is switch on at $9.00 \mathrm{am}$ and inner Relative humidity of the room is constantly maintain 45 \pm 5 .Recommended ASHRAE standard Relative humidity value for buildings in indoor is 45 to $55 \%{ }^{[2]}$ in India. . The experimental result obtained as per standard in the flow of $1400 \mathrm{cfm}, 2100 \mathrm{cfm}$ and $2700 \mathrm{cfm}$.

Carbon monoxide is not exceeding ASHRAE standard level in indoor environment ( 0 to $9 \mathrm{ppm}$ ) (parts /million) it is 0 in indoor all the time it should be same. Outdoor it should be $1 \mathrm{ppm}$ in morning peak hours in (9.00 am to $11.00 \mathrm{am})$ and remaining time $0 \mathrm{ppm}$.

$\mathrm{O}_{2}$ level in the room maintained $19 \pm 0.2$. Air velocity is 0.4 $\mathrm{m} / \mathrm{s}$ in middle position of the room in all the time it should be same one.

Thermal comfort is obtained from three modes of air flow in meeting hall for empty and partial load. But the human load increase to 45 occupants its obtained thermal comfort in $2100 \mathrm{cfm}$, and $2700 \mathrm{cfm}$. But human comfort not yet obtained in the three mode of air flow because of the obtained value of carbon dioxide. Without fresh air supply or ventilation the human comfort not yet obtained. From the experimental results the $2100 \mathrm{cfm}$ it's having comfort compare with $1400 \mathrm{Cfm}, 2700 \mathrm{Cfm}$.

\subsection{Thermal Comfort Index}

According to ASHRAE-55, thermal comfort has been defined as the condition of mind which illustrates satisfaction with the thermal environment, and thermal sensation is related to heat balance between the human body and its ambient thermal condition. Depending on the heat transfer, via heat gain or loss, the Thermoregulation system in a human brain regulates skin temperature to maintain a constant core body temperature of $36.5^{\circ} \mathrm{C}$. meanwhile indoor temperature $22^{\circ} \mathrm{C}$ to $26^{\circ} \mathrm{C}$, Relative humidity $42 \%$ to $48 \%$, Carbon monoxide 0 to $9 \mathrm{ppm}$, carbon dioxide 0 to $1000 \mathrm{ppm}$ and Oxygen $19.0 \pm 0.2 \%$ As per ASHARE. Thermal comfort index of the model are calculated using the fanger model. The volume of data obtained from the experimental value helps to find the comfort condition in high level accuracy.

The fanger model of thermal comfort is calculated by predicted mean vote and predicted percentage dissatisfied. The PMV and PPD model is based on the combined influence of relative humidity, air temperature, mean radian $\mathrm{t}$ temperature, air movement to that of clothing and activity level.

\section{$P M V=3.155\left(0.303 \mathrm{e}^{-0.114 M}+0.028\right) \mathrm{L}$ PPD = $100-95 \exp [-(0.03353$ PMV $4+0.2179$ PMV2 $)]$}

The experimental values are calculated to find the PPD and the results are been plotted below, to find the comfort condition. As per fanger model the thermal comfort index calculated. Normally the low bound that PMV $>0$ was recommended in summer and the up bound that $\mathrm{PMV}<0$ was recommended in winter.

\subsection{PMV-PPD model of cabin}

\begin{tabular}{|c|c|c|c|c|c|}
\hline $\begin{array}{l}\text { S.N } \\
\text { O }\end{array}$ & $\begin{array}{l}\text { AIR } \\
\text { FLOW } \\
\text { RATE }\end{array}$ & LOAD & $\begin{array}{l}\text { FRES } \\
\text { H AIR } \\
\text { SUPP } \\
\text { LY }\end{array}$ & $\begin{array}{l}\text { PMV (- } \\
3 \text { to }+3)\end{array}$ & $\operatorname{PPD}(\%)$ \\
\hline 1 & 1400 & $\begin{array}{l}\text { EMPT } \\
Y\end{array}$ & 10 & -0.46 & 9.5 \\
\hline 2 & 2100 & $\begin{array}{l}\text { EMPT } \\
Y\end{array}$ & 10 & $\begin{array}{l}. \\
.15\end{array}$ & 33.1 \\
\hline 3 & 2700 & $\begin{array}{l}\text { EMPT } \\
Y\end{array}$ & 10 & $\begin{array}{l}- \\
.15\end{array}$ & 32.6 \\
\hline 4 & 1400 & $\begin{array}{l}\text { P.LOA } \\
\text { D }\end{array}$ & 10 & $\begin{array}{ll}- & 0 \\
.38 & \end{array}$ & 8 \\
\hline 5 & 2100 & $\begin{array}{l}\text { P.LOA } \\
\text { D }\end{array}$ & 10 & $\begin{array}{l}- \\
.17\end{array}$ & 33.9 \\
\hline 6 & 2700 & $\begin{array}{l}\text { P.LOA } \\
\text { D }\end{array}$ & 10 & -18 & 34.5 \\
\hline 7 & 1400 & $\begin{array}{l}\text { F.LOA } \\
\text { D }\end{array}$ & 10 & 0.73 & 16.2 \\
\hline 8 & 2100 & $\begin{array}{l}\text { F.LOA } \\
\text { D }\end{array}$ & 10 & 0.15 & 5.5 \\
\hline 9 & 2700 & $\begin{array}{l}\text { F.LOA } \\
\text { D }\end{array}$ & 10 & 0.07 & 5.1 \\
\hline
\end{tabular}

\section{CONCLUSION}

Providing comfort environment inside the meeting hall is very complex because of subjective nature. This investigation presented the suitable comfort environment for human load. The results are presented in tabulation. The presented tabulation are expressed the temperature, relative humidity, carbon monoxide, carbon dioxide oxygen and relative velocity about variable loads, air flow rate, and fresh air supply. In general the value of $\mathrm{CO}_{2}$ in more compare 
from indoor to outdoor because of increasing human load. To summarize, from our experimental results $1400 \mathrm{cfm}$ for without human load, 1400cfm for partial load and 2100cfm for full load are the thermal comfort environment in meeting hall. The result shows that parameters are clearly. The carbon monoxide and oxygen and relative velocity are may be same because of observed values are same in maximum observation. But carbon dioxide temperature and relative humidity are changed in each and every frequency of variable climatic conditions.

As per fanger model the thermal comfort index calculated. Normally the low bound that PMV $>0$ was recommended in summer and the up bound that $\mathrm{PMV}<0$ was recommended in winter. Recommended ranges of indoor air design parameters were determined for meeting hall was $1400 \mathrm{cfm}$ air flow with $10 \%$ fresh air supplies for without load obtained PMV value -0.46 and PPD 9.1\%, 1400cfm air flow with $10 \%$ fresh air supply for Partial loads obtained PMV value -0.38 and PPD $8 \%$ and $2100 \mathrm{cfm}$ air flow with $10 \%$ fresh air supply for full loads obtained PMV value 0.15 and PPD 5.5\% are also the comfort environment as per thermal comfort index.

Concluding from these experimental results the quality of indoor air parameters are observed about variable climatic condition in normal standard fresh air supply. The experimental results and thermal comfort index are point out the comfort environment in cabin were both of the results are same, so on the predicted results are provide the human and thermal comfort with healthy environment. The results showed the PMV and PPD values are surprisingly closed to zero and the human and thermal comfort parameters are also the limit of ASHARE standard in the meeting hall.

\section{REFERENCES}

[1]. T.T.Chowa, K.F.Fong, B.Givoni, ZhangLin, A.L.S.Chan. Thermal sensation of Hong Kong people with increased air speed, temperature and humidity in airconditioned environment. Elsevier - Building and Environment (2010) Vol. 45 PP 2177 - 2183

[2]. Shubhajyoti Saha, Abhijit Guha, Subhransu Roy. Experimental and computational investigation of indoor air quality inside several community kitchens in a large campus. Elsevier-Building and Environment (2012) Vol.52 PP 177-190.

[3]. Mohammad Taleghani, MartinTenpierik, StanleyKurvers, Andyvanden Dobbelsteen. A review into thermal comfort in buildings Elsevier - Renewable and Sustainable Energy Reviews (2013) Vol. 26 PP 201-215

[4]. Fabrizio Ascione, Laura Bellia, Alfonso Capozzoli. A coupled numerical approach on museum air conditioning: Energy and fluid-dynamic analysis. Elsevier - Applied Energy (2013) Vol 103 PP 416-427

[5]. M. Kavgic, Mumovic, Z. Stevanovic, A. Young .Analysis of thermal comfort and indoor air quality in a mechanically ventilated theatre. Elsevier -Energy and Buildings (2008) Vol.40 PP 1334-1343

[6]. K. Luck. Energy efficient building services for tempering performance-oriented interior spaces - A literature review. Elsevier - Journal of Cleaner Production (2012) Vol.22 PP 1- 10

[7]. Li Lan, Li Pan, Zhiwei Lian, Hongyuan Huang, Yanbing Lin. Experimental study on thermal comfort of sleeping people at different air temperatures. Elsevier Building and Environment (2014) Vol.73 PP 24 -31

[8]. Frauke Oldewurtel, David Sturzenegger, Manfred Morari. Importance of occupancy information for building climate control. Elsevier-Applied Energy (2013) Vol.101 PP 521-53

[9]. Cuimin Li, Jianing Zhao. Experimental Study on Indoor Air Temperature Distribution of Gravity Air-Conditioning for Cooling. Elsevier - Energy Procedia (2012) Vol.17 PP $961-967$

[10]. Pawel Wargocki, David P. Wyon. Providing better thermal and air quality conditions in school classrooms would be cost-effective. Elsevier - Building and Environment (2013) Vol.59 PP 581- 589 